\title{
Nonparticipation of Older Adults in a Heart Disease Self-Management Project
}

Factors Influencing Involvement

\author{
JULIA A. DODGE \\ NOREEN M. CLARK \\ NANCY K. JANZ \\ JERSEY LIANG \\ M. ANTHONY SCHORK \\ University of Michigan School of Public Health
}

\begin{abstract}
Nonparticipation is an important issue in gerontological research. However, literature describing factors influencing participation of older adults in disease management programs is limited. This article contributes to the understanding of nonparticipation in older adults by reviewing the relevant literature and delineating a strategy for assessing the impact of nonparticipation on the generalizability of findings. Involvement in a study of an educational intervention for teaching self-management skills was investigated. Four hundred elderly cardiac patients were divided into three groups: participants in the self-management program evaluation; nonparticipants who declined involvement in the study, but agreed to provide a limited amount of information via telephone interview; and those who refused involvement of any kind. Participants were most likely to be male, with more formal education, who were more worried about their heart conditions, and less likely to perceive that help was available to them. Nonparticipants mentioned "time constraints," "transportation," and "physical limitations" as primary barriers to involvement.
\end{abstract}

Recent studies (Clark et al. 1992; Conger et al. 1987; Lorig, Lubeck, Kraines, Seleznick, and Holman 1985; Mullen, Laville, Biddle, and Lorig 1987) have demonstrated that education can enable older adults

AUTHORS' NOTE: The research reported in this article was supported by funds from the Henry J. Kaiser Family Foundation, Menlo Park, California, the National Heart, Lung, and Blood 
to manage better the health problems that assail them, and aid them to function more fully. To benefit, individuals must be willing to participate. Although it is unlikely that any one educational or service program will be universally appealing to older adults, planners of most interventions hope to attract as many participants as possible. There is a need to understand the factors that influence older adults to take part in service and education programs, and participate in studies to evaluate them.

There were two objectives in conducting the research reported here. The first objective was to determine if those who agreed to participate in the evaluation of a heart disease self-management program were representative of the larger population of older cardiac patients who were eligible to participate. The second objective was to determine why some declined to be involved so that a future project might be modified to appeal to a larger audience of elderly individuals.

\section{Nonresponse and Nonparticipation}

Most information on nonparticipation in the literature concerns bias in survey research and comes from studies of failure to respond to mailed or telephoned surveys, that is, not being available for interviews or not returning completed questionnaires.

Nonresponse has been shown to be greater for older than for younger adults (Annest and Mahaffey 1984; Kaye, Lawton, and Kaye 1990; O'Neil 1979; Thornberry 1987). Herzog and Rodgers (1988), for example, reported a linear decline in response rates with age in both sample surveys and panel resurveys. Older adults have also been shown to be more likely to refuse to participate in data collection efforts (DeMaio 1980; Napier 1962; Weaver, Holmes, and Glenn 1975). Brod and Feinbloom (1990) showed that requiring written consent reduces participation significantly. Groves (1989) states that the higher refusal rate among the elderly occurs across income level and ethnic and racial groups. 
Less formal education has been associated with nonparticipation. O'Neil (1979), in estimating the nonresponse bias due to refusals in telephone surveys, found the resistors to have less education. Groves, Miller and Cannell (1987) found that of those randomly selected to complete a telephone health survey, only $58 \%$ of those with less than a high sciool education consented as compared to $96 \%$ with at least 13 years of schooling.

The associations between nonparticipation and gender and race are less clear. Although a few studies have found nonparticipants in research studies more likely to be male (Groves 1989), other studies have not detected gender differences (Crique, Barrett-Connor, and Austin 1978; Kaye, Lawton, and Kaye 1990; Napier 1962; O’Neil 1979). In general, investigators have found lower refusal rates for Blacks (Hawkins 1975; O’Neil 1979; Weaver et al. 1975). However, the effects of gender, race, and education on participation of older adults have not been studied extensively.

Additional studies suggest that nonparticipants are sicker than participants. In a cardiovascular disease study involving interviews and lipid level testing over a 2-year period, Crique et al. (1978) found that the refusers were "less healthy," at least in terms of their past cardiovascular illnesses, although they had fewer risk factors. Janzon, Hanson, Isacsson, Lindell, and Steen (1986) found that men who rated their health status as "poor" were less likely to participate in a study of major health determinants in a population of 68-year-old men. Herzog and Rodgers (1988) suggested that health-related reasons may have accounted for a majority of nonresponses in older adults recruited for three different sample surveys. In a health-status survey, Rockwood, Stolee, Robertsen, and Shillington (1989) found that the cohort of elderly refusers over 75 years of age used significantly more medical services than did respondents, although this pattern was not found for those refusers 65 to 74 years of age.

Following a review of participation of older adults in both health service programs and research, Carter, Elward, Malmgren, Martin, and Larson (1991) concluded that it is no less feasible to include adults up to age 74 in surveys, clinical research, and health promotion programs. Older adults, they state, are good study participants who comply with study protocols and the quality of their participation is comparable to or only modestly lower than other age groups. 
In sum, however, these studies do not tell us as much as researchers need to know about older nonparticipation in either service programs or research. They provide little information about gender differences, and even less about nonparticipation beyond basic sociodemographic characteristics. Further, information is particularly limited related to projects where the burden of participation is heavier than simply responding to survey forms or telephone interviews.

\section{Participation in Evaluation Research}

Nonresponse rates in survey research introduce bias into survey estimates because the logic of the process demands that attitudes, knowledge, and behavior of the sample be attributable to the population in question. The sample must be externally valid, that is, a mirror image of the larger population on important dimensions, generally sociodemographic. In survey research, nonresponse reduces the sample's representativeness.

Participation in evaluation of health service programs, particularly health promotion and education, must be thought of somewhat differently than participation in conventional surveys. Generally, the demands on participants are greater in evaluation research. The selfmanagement program in which subjects in the study presented here were asked to participate, required willingness to travel to a local hospital on four occasions to meet with 8 to 10 other individuals in group sessions 2 hours in length. In addition, participants had to agree to respond to questionnaire items during 1-hour-long telephone interviews conducted on five separate occasions over an 18-month time period.

A potential enrollee in such a program may or may not participate for several reasons. For example, the instructional methods may not appeal to a particular individual. Learning styles vary (Srinavasan 1977), are difficult to classify and predict, and are not necessarily associated with the sociodemographic or other structural variables used to determine representativeness of samples (DiPietro and Clark 1983). Participants in a program are more likely to have agreed to be involved if they perceived a need and/or value in the program content, and the program format is an acceptable pattern for learning. 
For some, participation in a program places a burden due to the time and/or travel involved. Each program will attract only those individuals willing to assume the burden. Further, the data collection activities of program evaluations, just as in survey research, will discourage participation by some individuals.

Green and Figa-Talamanca (1974) have suggested that in program evaluation internal validity (that is, the program indeed produced the results observed) is, in the long run, more critical than external validity. This view is held precisely because few programs will appeal universally to the eligible audiences and enrollment in health education and service programs is voluntary. At the same time, Green and FigaTalamanca (1974) and others (Windsor, Baranowski, Clark, and Cutter 1984) have noted that health programs must reflect economy of scale, that is, they must involve sufficient numbers of people to justify the costs. External validity is important because one wishes to know if those agreeing to participate in a program are like the larger target population on some critical dimensions assuming that (a) the program will be more appealing to individuals fitting that profile and (b) replication of it will attract an audience of sufficient size to warrant the costs. As little information is available about participation of older adults in health promotion and education and in studies evaluating such programs, the investigators were interested in knowing how similar the profiles of participants, nonparticipants, and refusers of an evaluation of a heart disease self-management program were to each other. Participants were defined as those who agreed to take part in all aspects of the program and its evaluation. Nonparticipants were those who declined involvement in the program and its evaluation but agreed to provide some data to the researchers including why they chose not to participate. Refusers were those who declined involvement of any kind. Identifying the differences among these groups seemed a profitable route to discovering ways in which future projects might be designed to engender more participation.

\section{Study Method}

The data on nonparticipation reported here were collected as part of a larger study to evaluate the effectiveness of a heart disease 
self-management education program, entitled "take PRIDE," for older adults with heart disease (hereafter referred to as the parent study). Take PRIDE is an interactive educational program comprising four 2-hour group meetings of $\mathbf{8}$ to $\mathbf{1 0}$ older adults with diagnosed heart disease. Based on social cognitive theory, particularly principles of self-regulation, the program introduces participants to a self-regulatory process for identifying and resolving problems they confront in managing their heart conditions. Vehicles for teaching include a videotape, workbook, and instruction from a health educator who also functions as the group facilitator. PRIDE is an acronym for phrases that reflect the self-regulatory steps participants are asked to follow: problem selecting, researching one's daily routine, identifying a behavioral goal, developing a plan to reach one's goal, and establishing a reward for making progress. The program is designed so that participants can work individually on their particular management problem (e.g., diet, exercise, symptom experience, medicine taking) and, at the same time, receive help and social support from group members.

Potential participants in the parent study were identified through a review of medical records at cardiac outpatient facilities in three large hospitals in southeastern Michigan. All potential subjects had to meet the following inclusion criteria: 60 years of age and over, diagnosed cardiac disease, treated daily by at least one heart medication, and seen by a physician at least once every 6 months. Cardiac disease was defined as any condition involving the heart itself (e.g., arrhythmia, angina, myocardial infarction, or valvular disease). Patients were excluded if hypertension was their only heart-related diagnosis, because hypertension is most often asymptomatic. The intent of the program was to attract participants limited by their heart disease on a daily basis. Length of time since initial diagnosis ranged from a minimum of 6 months to 20 or more years.

Potential subjects received an introductory letter explaining that they had been referred to the program by their physicians, followed by a telephone contact describing the study and inquiring about their interest in participation. A script was developed to structure the telephone contact and to insure that all potential participants received the same information regarding the study. Individuals were told that the purpose of the study was to learn more about the ways people with a heart condition manage their daily routine. Each potential subject 
was told he or she would be interviewed by telephone a total of five times over 18 months and would be asked questions about his/her health and how he or she had been managing the heart regimen. In addition, they were told that they might be offered the opportunity to attend an educational program focusing on management of their health problems. The time commitment and format of the group meetings were explained. First, potential participants were told that the educational program consisted of four 2-hour sessions held weekly at their local hospital. In addition, they were informed that the classes would be attended by 8 to 10 older adults experiencing different types of heart conditions and that a health educator would lead the classes. The focus of the classes would be looking at new ways to better manage the heart condition with an opportunity to share ideas with other group members. An individual's decision regarding participation in the study was made prior to knowing whether or not he or she would be asked to attend the educational sessions. Once agreements to participate were obtained, baseline interviews were conducted.

To carry out the study reported here, that is, to further understand the characteristics of nonparticipation, attempts were made to interview all individuals declining participation in the parent study during a specific 8-month time period (July 1989 to February 1990). A 15-minute telephone interview was developed that included items assessing the following: sociodemographic characteristics, perceptions of health-related quality of life, use of health care services, existing comorbidities, and reasons for electing not to participate in the self-management program evaluation. A list of frequently described impediments was read to those willing to complete the nonparticipant telephone interview and respondents were asked to indicate whether or not each impediment influenced their decision not to join the parent study. Individuals were then asked to rank the factors contributing to their decision in order of importance to them.

A total of 560 persons who met the criteria and could be contacted by telephone were invited to enroll in the parent study. Three groups emerged: participants-279 (51\%) who agreed to participate in all aspects of the self-management education study, nonparticipants$121(21 \%)$ who declined participation but agreed to respond to the nonparticipant telephone interview, and refusers-160 (28\%) who refused all involvement. 
Data on the refusers were limited to the initial reason given the interviewer for refusing to join the study and those variables available on physician referral forms: sex, age, and the criteria used in the original screening process. The more extensive data from participants and nonparticipants could be compared across three major sets of variables: sociodemographic characteristics, health-related qualityof-life variables, and use of health care services. To assess whether participants and nonparticipants could be differentiated by these variables, stepwise discriminant analyses were completed. This procedure generated the constellation of variables that best distinguished sets of subjects, that is, participants from nonparticipants. Additional analyses identified specific reasons for refusal overall. Preliminary comparisons of participants, nonparticipants, and refusers for potential discriminating variables were completed using chi-square, the analysis of variance, and $t$ tests as appropriate.

\section{Results}

\section{SOCIODEMOGRAPHIC CHARACTERISTICS}

Of the 560 subjects between the ages of 60 and 92 years contacted for the parent study, 303 were males and 257 were females. Of the males, $52 \%$ agreed to participate compared to $47 \%$ of the females. Of those who decided not to enroll in the parent study, women were significantly more likely than men to agree to complete the nonparticipant telephone interview. In addition, participants in the parent study were younger (60 to 69 years) and more educated. Refusers, when compared to the other two groups were more often male and over 70 years of age. When compared to the participant group, nonparticipants were significantly more likely to be living in the same household as their children (see Table 1).

\section{HEALTH-RELATED QUALITY-OF-LIFE VARIABLES}

Responses to nine quality-of-life measures (six related to patient perceptions regarding the impact of their heart conditions on the quality of their daily lives, one overall rating of health, and two social 


\section{TABLE 1}

Age and Family Living Characteristics of Participants,

Nonparticipants, and Refusers $(N=560)$

\begin{tabular}{|c|c|c|c|c|c|c|}
\hline \multirow[b]{2}{*}{ Characteristic } & \multicolumn{2}{|c|}{ Participants $(\mathrm{n}=279$ ) } & \multicolumn{2}{|c|}{ Nomparticipants $(\mathrm{n}=121)$} & \multicolumn{2}{|c|}{ Refusers $(\mathrm{n}=160)$} \\
\hline & $x$ & SD & $\bar{x}$ & SD & $\mathbf{x}$ & SD \\
\hline Age* & 68.8 & 6.5 & 70.4 & 7.1 & 73.6 & 6.9 \\
\hline $\begin{array}{l}\text { Children in same } \\
\text { household** }\end{array}$ & 0.21 & 0.49 & 0.34 & 0.67 & - & - \\
\hline
\end{tabular}

*Significant difference by ANOVA at $p<.01$; **Significant difference by $t$ test at $p<.05$.

comparison items) were available for analysis. Both participants and nonparticipants when asked "How would you rate your present health?" on a 5-point scale ranging from poor to excellent, tended to respond that it was "good" or "fair." When specifically asked how they viewed their heart condition, nonparticipants were significantly more likely to say that it was "not serious at all" and that it "does not interfere at all" with daily life. Although perceptions of overall health status were similar for males and females, significant within sex differences emerged in the perceptions of the heart conditions. Female nonparticipants were significantly more likely than female participants to say they had "much difficulty" or "could not do daily activities" because of the heart problem. When asked the degree to which their heart conditions interfered with their lives, female nonparticipants were also more likely than female participants to give responses at the extremes of the scale, that is, either the condition interfered "a great deal" or "not at all." Although these responses may seem contradictory, they may represent two subgroups of nonparticipating women: those feeling too well to be interested in a study involving attendance at a health education program, and those physically unable to participate. In addition, nonparticipants were much more likely than participants to report that in the past 4 weeks they had someone available to help them "as much as needed."

\section{HEALTH CARE USE}

Data on six health-care use measures were available (three regarding the total number of outpatient visits, emergency room visits, and 
TABLE 2

Demographic, Quality-of-Life, and Health Care

Use Variables Used in Discriminant Analysis

\begin{tabular}{ll}
\hline Category & Variable \\
\hline Demographics & Sex \\
& Age \\
& Race \\
& Education \\
& Marital status \\
& Employment status \\
& Rating of present health \\
Quality of life & Health compared to others \\
& Perceived seriousness of heart condition \\
& Worry regarding heart condition \\
& Interference of heart condition with life in general \\
& Difficulty with daily activities because of heart condition \\
& Interference of heart condition with family and friends \\
& Manage heart condition compared to others \\
& Amount of help available \\
Total number outpatient visits (doctor of medicine/clinic) & Total number emergency room visits \\
in past 6 months & Total number hospitalizations \\
& Total number outpatient visits due to heart condition \\
& Total number emergency room visits due to heart condition \\
& Total number hospitalizations due to heart condition \\
\hline
\end{tabular}

hospitalizations and three regarding use of such services specific to one's heart condition). During the previous 6 months, nonparticipants had significantly more outpatient visits (mean $=5.2$ versus $4.1, p=$ .009) than participants, but did not differ in the number of emergency room visits or hospitalizations. No differences were found between participants and nonparticipants regarding use of any health care services specific to the heart condition.

\section{DISCRIMINANT ANALYSIS}

To determine if a set of sociodemographic characteristics, qualityof-life variables, and health care use measures could classify those who elected to participate in the parent study, a discriminant analysis was undertaken. Table 2 lists the specific content variables within each 
TABLE 3

Summary of Correct Classification Rates of Discriminant Analysis Models to Identify Variables Distinguishing Participants from Nonparticipants

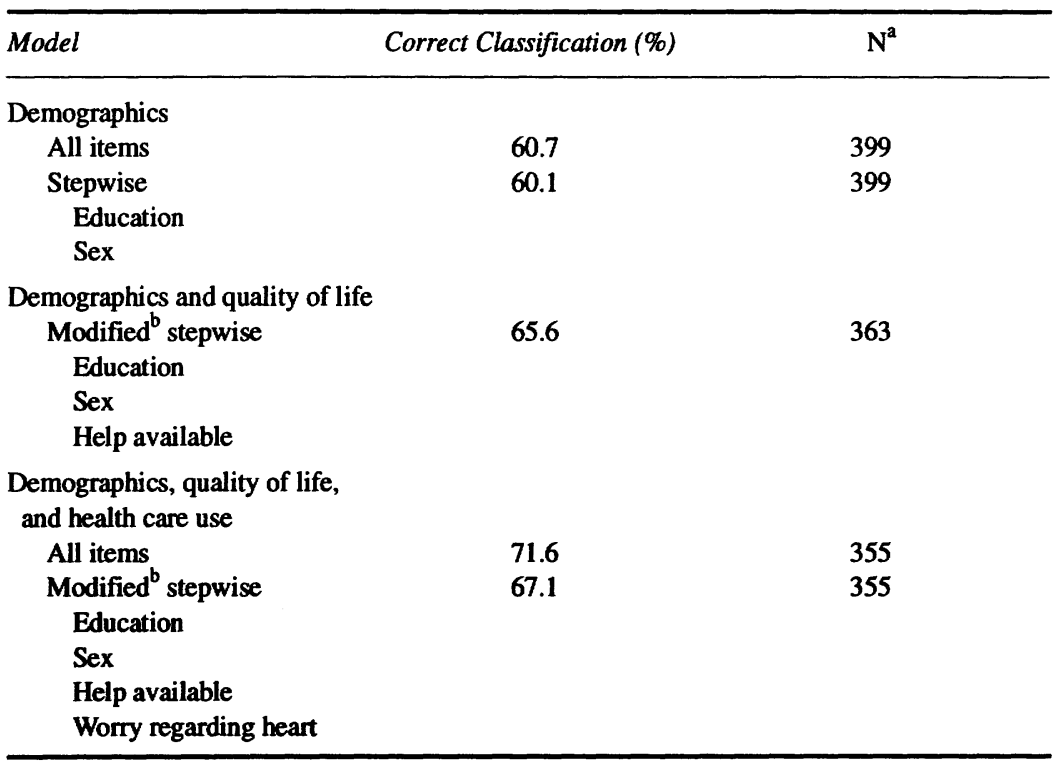

a. Sample size varies due to missing data for certain variables.

b. Modified indicates that sex and education are forced into the model prior to the search for additional predictors.

category. Table 3 contains a summary of the discriminant analysis models.

Adiscriminant analysis using all sociodemographic items produced a model where $60.7 \%$ of the sample could be correctly classified as participants or nonparticipants. When a forward stepwise discriminant analysis was employed, level of education and sex were significant $(p<$ 0.05 ) and correctly classified $60.1 \%$ of the sample. Those choosing to participate were more likely to be educated and male.

To determine whether quality-of-life variables could improve prediction above what could be accomplished by sociodemographic identification, a modified forward stepwise discriminant analysis was completed forcing education and sex into the model. The "availability of help" variable was selected and improved the classification rate of the model from $60.1 \%$ to $65.6 \%$ (i.e., males with higher levels of education and less help at home were more likely to agree to participate). 
When the health care use variables were added to a stepwise model that forced inclusion of the education and sex variables, no improvement in classification was achieved (59.7\%).

Finally, to determine a concise and parsimonious model, a discriminant analysis containing all items was undertaken and $71.6 \%$ were correctly classified. This represents a marginally useful classification. An efficient and easily determined model $(67.1 \%)$, discerned by the forced stepwise approach, contains four measures (level of education, sex, worry about heart condition, and perceived availability of help). Those who were most likely to participate in the study were more educated, male, more worried about their heart condition, and less likely to perceive that help was available.

The final phase of the analysis examined the reasons given for declining participation in the program evaluation. First, all nonparticipants (including the refusers) were asked to briefly state their reasons for declining participation (Table 4). Those refusing all involvement were more likely to say they were "not interested," had "physical limitations," and "perceived absence of a heart problem." Nonparticipants (those declining the participation but agreeing to provide data) most often initially volunteered "time constraints," "transportation," and "physical limitations" as barriers to their participation. Of the 33\% who mentioned being physically unable to participate, only one third said the limitation was due to a heart condition. Overall, nonparticipants' rank order of these reasons were as follows: transportation, time constraints, and lack of difficulty managing the medical regimen. Second, to further refine the understanding of likely barriers to participation, a list of possible barriers was read to those nonparticipants who completed the telephone interview. If a specific factor influenced their decision not to join the study, they were asked to rank order the three most important factors. In addition, $35 \%$ of nonparticipants responded yes to the statement that they preferred not to participate in research projects.

Table 5 illustrates that gender differences were found in the reasons given for nonparticipation. Overall, female nonparticipants were significantly more likely than male nonparticipants to mention transportation, "weather," "feeling physically unable," and "distance to the program sessions" as barriers to participation. When asked to select the primary barrier, women were more likely to select transportation 
TABLE 4

Nonparticipants and Refusers:

Reasons Given for Declining Participation $(N=281)$

\begin{tabular}{lcc}
\hline Reason & \% Nonparticipants $(\mathrm{n}=121)$ & \% Refusers $(\mathrm{n}=160)$ \\
\hline No concerns with heart problems & 10.1 & 4.4 \\
No heart problem & 0 & 13.8 \\
Physically unable & 16.0 & 20.1 \\
Transportation & 21.0 & 6.3 \\
Distance to classes & 2.5 & 0.0 \\
Need to care for others & 5.9 & 3.8 \\
Time commitment & 29.4 & 3.8 \\
Dislike groups & 1.7 & 0.0 \\
Not interested & 13.4 & 47.8 \\
\hline
\end{tabular}

TABLE 5

Nonparticipants: All Factors Contributing to Nonparticipation by Sex

\begin{tabular}{lrc}
\hline Reason & \% Male $(\mathrm{n}=50)$ & \% Female $(\mathrm{n}=71)$ \\
\hline Transportation & 10.2 & $60.6^{*}$ \\
Weather concerns & 8.2 & $56.3^{*}$ \\
Time commitment & 56.0 & 46.5 \\
Physically unable & 18.4 & $43.7^{*}$ \\
Dislike research & 33.3 & 35.7 \\
Distance to classes & 8.2 & $31.0^{*}$ \\
Need to care for others & 16.3 & 18.3 \\
Work conflict & 20.4 & 11.3 \\
\hline
\end{tabular}

*Differences significant by $t$ test at $p<.01$.

or being physically unable, whereas men chose "time commitment" or "conflicts with work."

\section{Discussion}

This systematic attempt to explore involvement in an evaluation of self-management education for elderly heart patients has produced useful information about the target population. Participants were more likely to be better educated, male, more worried about their illness, 
and to have less help available at home than nonparticipants. Nonparticipants, that is, those who declined full participation but agreed to be interviewed to discuss why, overall believed their heart condition to be less serious. However, within that group, women who declined to participate anchored a continuum. At one end were women who believed their heart condition was not a problem and did not interfere with daily life, and at the other end were women who believed themselves too ill to take part. Women did report significantly more arthritis, walking problems, and back pain. Therefore, it is quite likely that a subset of women were too ill to participate because they experienced these comorbidities. Nonparticipants also lived in situations where their children were present and reported more often that help was available if needed.

The least is known about those who refused even to provide any data on nonparticipation. They were significantly older males who expressed little interest in this type of project, although their health care providers believed they could benefit from it. A small number of refusers believed themselves too physically limited to participate.

Study findings comprise information useful to clinicians providing educational programs to older adults as well as to researchers evaluating programs. The four variables identified in the most efficient discriminant analysis model are factors that can be quickly assessed in the clinical setting. Two of these variables, education and sex, are not alterable. However, health care professionals could determine the level of worry by an older patient regarding their heart condition and the amount of help perceived to be available from others. Worried individuals with inadequate assistance at home could be priority clients for group-oriented education and support programs, and study results suggest that these individuals find self-management programs attractive. Such programs can have positive impact on a patient's level of worry, can provide social support and strategies to help older adults compensate for lack of help at home (Clark et al. 1991), and are a realistic investment of time and energy for these patients.

Findings from this study of nonparticipation also suggest that alternative approaches are needed to involve greater numbers of older adults in evaluation research. Two types of strategies can be envisioned. One type concerns recruiting for participation more people from among the cohort predisposed toward research in general, and 
group format programs such as the self-management one described here. The other type concerns alternative ways to provide education and service to reach those not attracted to or able to attend group meetings.

To recruit more older individuals from among those who would consider participation in a group-format program and research to evaluate it, several approaches may be needed. Additional counseling may be necessary when a health care professional identifies an older adult who is "undermanaging" his or her health problem and does not recognize that this is the case. Such patients may also not perceive their condition as serious. Older males may particularly fall into this category. Perceptions of seriousness of illness have frequently been shown to be a determinant of health behavior (Becker and Rosenstock 1987). Counseling could both emphasize the seriousness of the health problem and the potential for education or service to promote fuller functioning. For those patients who believe themselves too ill to participate, special encouragement and particularly reassurance from the health care provider may be helpful. According to the study presented here, individuals who feel incapacitated are more likely to be women and efforts to reduce these feelings among older female patients are warranted. For individuals who face time and transportation difficulties in attending a program involving several meetings held at a location some distance from them, condensed versions of the program offered at alternate sites might be explored. In the parent study, cab fare was offered to offset transportation costs to reach the site of the self-management education. However, for many nonparticipants this option did not resolve their fears about travel or concerns regarding the time entailed.

Providing alternative formats could be a way to reach more older adults with education and service. For example, for individuals disinterested in leaving home or too physically limited to travel to a program, videotapes or easy-to-use large-print self-instruction materials may be useful. Another strategy may be to provide a one-session program tailored to their needs during the hospital stay or in conjunction with a home visit.

Nonparticipants in our study had somewhat less formal schooling than participants, and these individuals likely need both special encouragement from their health care providers to participate in group 
programs and alternative formats; formats that place few demands in terms of literacy and medical terminology.

Particular attention to needs of older women seems warranted from the findings. Formats that recognize their more frequent reports of comorbidity are needed, as is special focus on their perceptions of their illness as serious and highly intrusive.

When it comes to providing the data needed to evaluate health education and service programs, the older adults in the research discussed here were, in the main, both willing and able. Participants were interviewed in lengthy telephone conversations on several occasions over an 18-month period. One third of potential study enrollees were refusers who were completely resistant to data collection. For the most part, these tended to be older men disinterested in the research. However, two thirds of nonparticipants were not opposed to participating in research and all were willing to provide some data to interviewers. The telephone proved an efficient and acceptable vehicle for data collection. As noted by others (Herzog and Rodgers 1988), this method of data collection is convenient and nonobtrusive to all but those with hearing impairments or who do not have access to a telephone. Colombotos (1969) has also shown telephone data to be comparable to those collected face-to-face. Although it was beyond the scope of this study to compare participation when data were collected by telephone with other means, the method proved highly productive for the research described.

As no one health program appeals to all eligible individuals, and some are simply disinterested in research, the participation of approximately half of the older individuals invited to take part in the program evaluation reported here seems consistent with findings of other investigations of older populations (Herzog and Rodgers 1988). In addition, the sample for the parent study appears to be fairly representative on sociodemographic dimensions of the larger population eligible to participate, being only slightly younger and better educated. Further, the program appears to have reached individuals who appear to need help-those with limited social support (fewer adult children in the same household and fewer sources of help available when needed). However, to have a stronger impact on the morbidity rates evident in the cohort of older adults with heart disease, those declining participation must also be reached. This study has described some 
salient features of these individuals and has suggested some ways in which they might be better served in turn and contribute to evaluation research.

\section{REFERENCES}

Annest, Joseph L. and Kathryn Mahaffey. 1984. "Blood Lead Levels for Persons Ages 6 Months - 74 Years: United States, 1976-80.” In Vital and Health Statistics (series 2). National Health and Nutrition Examination Survey no. 233 (DHS publication no. PHS 84-1983). Hyattsville, MD: U.S. Department of Health and Human Services.

Becker, Marshall H. and Irwin M. Rosenstock. 1987. "Comparing Social Learning Theory and the Health Belief Model." Advances in Health Education and Promotion 2:245-49.

Brod, Meryl S. and Richard I. Feinbloom. 1990. "Feasibility and Efficacy of Verbal Consents." Research on Aging 12(3):364-73.

Carter, William B., Kurt Elward, Judith Malmgren, Mona L. Martin, and Eric Larson. 1991. "Participation of Older Adults in Health Programs and Research: A Critical Review of the Literature." The Gerontologist 31(5):584-92.

Clark, Noreen M., Marshall H. Becker, Nancy K. Janz, Kate Lorig, William Rakowski, and Lynda Anderson. 1991. "Self-Management of Chronic Disease by Older Adults: A Review and Questions for Research." Joumal of Aging and Health 3(1):3-27.

Clark, Noreen M., Nancy K. Janz, Marshall H. Becker, M. Anthony Schork, John Wheeler, Jersey Liang, Julia A. Dodge, Steven Keteyian, Ken L. Rhoads, and John T. Santinga. 1992. "Impact of Self-Management Education on the Functional Health Status of Older Adults with Heart Disease." The Gerontologist 32(4):438-43.

Colombotos, John. 1969. "Personal Versus Telephone Interviews: Effect on Responses." Public Health Reports 84:773-82.

Conger, Beach, Eugene C. Nelson, Allen J. Dietrich, Chase Blanchard, Gregory J. McHugo, Jeannette J. Simmons, Pamela Vernon, Roger Secker-Walker, and John H. Wasson. 1987. "Effectiveness of Physician Antismoking Advice." American Journal of Preventive Medicine 3(4):223-28.

Crique, Michael H., Elizabeth Barrett-Connor, and Melissa Austin. 1978. "Differences Between Respondents and Non-Respondents in a Population-Based Cardiovascular Disease Study." American Journal of Epidemiology 108:367-72.

DeMaio, Theresa J. 1980. "Refusals: Who, Where and Why." Public Opinion Quarterly 44:223-33.

DiPietro, Rocco and Noreen Clark. 1983. “Adolescents' Communication Styles for Learning About Birth Control From Mass Media." Health Education Quarterly 10(2):106-19.

Green, Lawrence W. and Irene Figa-Talamanca. 1974. "Suggested Designs for Evaluation of Health Education Programs." Health Education Monographs 2:34-60.

Groves, Robert M. 1989. Survey Errors and Survey Costs. New York: Wiley.

Groves, Robert M., Peter V. Miller, and Charles F. Cannell. 1987. "Differences Between the Telephone and Personal Interview Data." In Vital and Health Statistics. National Center for Health Statistics, technical series 2, no. 106 (DHS Publication no. PHS 87-1380). Hyattsville, MD: U.S. Department of Health and Human Services.

Hawkins, Darnell F. 1975. "Estimation of Nonresponse Bias." Sociological Methods and Research 3:461-85. 
Herzog, A. Regula and Willard L. Rodgers. 1988. "Age and Response Rates to Interview Sample Surveys." Journal of Gerontology: Social Sciences 43:S200-05.

Janzon, Lars, Bertil S. Hanson, Sven-Olof Isacsson, Sven-Eric Lindell, and Bertil Steen. 1986. "Factors Influencing Participation in Health Surveys: Results From Prospective Population Study 'Men Born in 1914' in Malmö Sweden." Journal of Epidemiology and Community Health 40:174-77.

Kaye, Janet M., Powell Lawton, and Donald Kaye. 1990. “Attitudes of Elderly People About Clinical Research on Aging." The Gerontologist 30:100-06.

Lorig, Kate, Deborah Lubeck, R. Guy Kraines, Mitchell Seleznick, and Halsted R. Holman. 1985. "Outcomes of Self-Help Education for Patients With Arthritis." Arthritis Rheum 28(6):680-85.

Mullen, Patricia D., Elizabeth A. Laville, Andrea K. Biddle, and Kate Lorig. 1987. "Efficacy of Psychoeducational Interventions on Pain, Depression, and Disability in People with Arthritis: A Meta-Analysis." Jourmal of Rheumatology Suppl. 15:33-39.

Napier, John A. 1962. "Field Methods and Response Rates in the Tecumseh Community Health Study." American Journal of Public Health 52:208-16.

O'Neil, Michael J. 1979. "Estimating the Nonresponse Bias Due to Refusals in Telephone Surveys." Public Opinion Quarterly 43:218-32.

Rockwood, Kenneth, Paul Stolee, Duncan Robertsen, and E. Richard Shillington. 1989. "Response Bias in a Health Status Survey of Elderly People." Age and Aging 18:177-82.

Srinavasan, Lyra. 1977. Perspectives on Nonformal Adult Learning. Boston, MA: World Education.

Thornberry, Owen T. 1987. "An Experimental Comparison of Telephone and Personal Health Interview Surveys." In Vital and Health Statistics. National Center for Health Statistics, technical series 2, no. 106 (DHS publication no. PHS 87-1380). Hyattsville, MD: U.S. Department of Health and Human Services.

Weaver, Charles N., Sandra L. Holmes, and Norval D. Glenn. 1975. "Some Characteristics of Inaccessible Respondents in a Telephone Survey." Journal of Applied Psychology 60:260-62.

Windsor, Richard, Thomas Baranowski, Noreen Clark, and Gary Cutter. 1984. Evaluation of Health Promotion and Education Programs. Palo Alto, CA: Mayfield.

Julia A. Dodge is Research Assistant in the School of Public Health at the University of Michigan.

Noreen M. Clark is Professor and Chair in the Department of Health Behavior and Health Education in the School of Public Health at the University of Michigan. Her research interests are self-regulation and self-management of chronic disease.

Nancy K. Janz is Assistant Professor in the Department of Health Behavior and Health Education in the School of Public Health at the University of Michigan.

Jersey Liang is Professor and Research Scientist in the Department of Health Behavior and Health Education in the School of Public Health at the University of Michigan.

M. Anthony Schork is Professor in the Department of Biostatistics at the University of Michigan. 